\title{
TRIF, and TRIF-Interacting TLRs Differentially Modulate Several Adenovirus Vector-Induced Immune Responses
}

\author{
D.M. Appledorn ${ }^{\mathrm{a}} \quad$ S. Patial ${ }^{\mathrm{b}} \quad$ S. Godbehere ${ }^{\mathrm{a}} \quad$ N. Parameswaran $^{\mathrm{b}} \quad$ A. Amalfitano ${ }^{\mathrm{a}}$
}

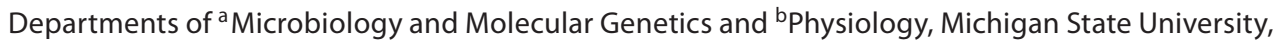
East Lansing, Mich., USA

\section{Key Words}

Adenovirus $\cdot$ Vaccine $\cdot$ Gene therapy $\cdot$ Antibodies .

Inflammation $\cdot$ Signal transduction $\cdot$ TRIF $\cdot$ TLR

\begin{abstract}
The use of Adenovirus (Ad)-based vectors has proven to be a useful platform for the development of gene therapy and vaccine protocols. The immunological mechanisms underlying these properties need to be identified and understood to foster safer, more efficacious use of this important gene transfer platform. Our recent studies have confirmed an important role for MyD88 dependent toll-like receptor (TLR) pathways as mediators of these responses. In this study, we confirm that TLR3, TLR4 and TRIF (TIR-domain-containing adapter-inducing interferon- $\beta$ ) can also have augmentative or inhibitory roles during Ad-induced immune responses. Importantly, our studies revealed that TLR4 acts to suppress several aspects of the Ad-induced innate immune response, a finding not previously reported for this TLR in any model system. In addition, using MyD88 and TRIF double knockout mice, we demonstrate that the MyD88 and TRIF adaptor proteins can play either additive or redundant roles in mediating certain aspects of Ad vector-induced innate and adaptive immune responses. Furthering this complexity, our model system strongly suggests that non-TLR based systems must not only exist, but also have a significant role to play during Ad vector-mediated induction of adaptive immune responses.

Copyright $\odot 2009$ S. Karger AG, Basel
\end{abstract}

\section{Introduction}

Adenoviruses (Ads) are considered one of the most promising platforms for both gene therapy and vaccine development, which is demonstrated by the fact that the vast majority of clinical trials utilize Ads for these types of purposes. However, the innate inflammatory response induced by Ad-mediated gene delivery is a complex phenomenon. This complexity may limit the potential utility of Ad vectors in certain clinical settings, such as gene therapy, while augmenting their usefulness for other applications, such as vaccines. Recently, we and others have begun to identify the molecular mechanisms underlying Ad induction of innate and adaptive immune responses associated with each of these applications. Specifically, we have identified a role for the complement system, including both classical and alternative arms, in Ad-mediated inductions of thrombocytopenia, the release of systemic cytokines and chemokines, as well as the dramatic gene dysregulation in the murine liver following intravenous virus delivery [1-4]. In addition to those responses, we and others have also delineated a significant role for toll-like receptors (TLRs) and/or TLR adaptor proteins in mediating Ad-induced innate and adaptive immune responses [5-12].

TLRs are type 1 transmembrane pathogen recognition receptor proteins that contain leucine-rich repeat extracellular domains and a cytosolic toll-interleukin-1 receptor (TIR) homology domain that is essential for intra-

\section{KARGER}

(ㄷ) 2009 S. Karger AG, Basel

Fax +41613061234 E-Mail karger@karger.ch www.karger.com www.karger.com/jin
Dr. Andrea Amalfitano

Department of Microbiology and Molecular Genetics, Michigan State University 4194 Biomedical and Physical Sciences Bldg.

East Lansing, MI 48823 (USA)

Tel. +1 517884 5324, Fax +1 517353 8957, E-Mail amalfit1@msu.edu 
cellular signaling. Upon activation, TLRs recruit TIR containing adaptor molecules, including myeloid differentiation primary response gene 88 (MyD88), TIR-domain-containing adapter-inducing interferon- $\beta$ (TRIF, also known as TICAM1), TIR domain-containing adaptor protein (TIRAP, also known as MAL), and TRIF-related adaptor molecule (TRAM, also known as TIRP). Many studies of TLR signaling have utilized MyD88 knockout mice, since MyD88 is an essential signaling component for all TLRs except TLR3 [reviewed in 13]. Signaling through MyD88 leads to the degradation of I $\mathrm{B} \alpha$ and nuclear translocation of NFKB, as well as activation of the mitogen-activated protein kinase (MAPK) pathways. As a result, IL-6, IL-1 $\beta$, TNF- $\alpha$ and IL-12(p40) are produced through MyD88-mediated signaling. Activation of some TLRs, such as TLR3 and TLR4, can occur independently of MyD88. The adaptor molecule TRIF is essential for MyD88-independent signaling through these receptors [13]. Signaling through TRIF can also activate $\mathrm{NF \kappa B}$, leading to the production of proinflammatory cytokines. TRIF signaling also leads to the phosphorylation of IFN regulatory factor 3, which results in type I IFN induction [reviewed in 14]. Studies using MyD88 and TRIF knockout mice have also demonstrated a critical role for these 2 adaptor proteins in mediating TLR signaling in a temporal manner. That is, MyD88 is required for early signaling events while TRIF is required for late signaling events $[15,16]$.

In studies evaluating the role of TLR pathway signaling in Ad-specific inductions of innate and adaptive immunity, we and others have identified several TLR proteins that partially contribute to the rapid response to systemically delivered Ad-based vectors. Specifically, we have shown that mice genetically deficient in MyD88 have: (1) dramatically altered cytokine and chemokine responses; (2) significantly altered MAPK/NFкB activation kinetics; (3) altered liver transcriptome profiles, and (4) reduced adaptive immune responses subsequent to treatment with an Ad vector expressing an immunogenic transgene in vivo [8]. Furthermore, we recently reported that TLR2 is important for Ad-induced NFKB and MAPK activation, cytokine and chemokine release, and humoral immunity in vivo [5]. In addition to our work, other groups have also revealed the role of TLR9, IL-1R (a MyD88 utilizing receptor) and type I interferons in many of these responses both in vitro and in vivo $[6,9-$ $12,17]$.

Our previous work in vitro, has also indicated that TRIF is required for Ad-induced innate immune responses [8]. In the current study, we expand upon this observation through the use of mice individually deficient in TRIF, TLR3 or TLR4 genes, or genetically deficient in both the MyD88 and TRIF genes. As a result, we have identified that through TRIF, both TLR3 and TLR4 partially modulate Ad-induced innate and humoral adaptive immune responses to Ad-based vectors, as well as the transgene the vector expresses. Furthermore, we demonstrate that robust humoral responses to the Ad vector, or the transgene it expresses, can persist despite loss of MyD88- and TRIF-dependent signaling. These studies suggest that specific manipulations of TLR pathways that require MyD88 or TRIF may significantly alter aspects of the adaptive immune response to Ad-based vectors, but that redundant pathogen sensing systems must also exist that, in some instances, compensate for the loss of MyD88 and/or TRIF mediated functions. The implications of these important findings are discussed.

\section{Materials and Methods}

Adenovirus Vector Production and Characterization

The recombinant Ad vector, [E1-]Ad5-LacZ is a vector which carries a CMV-LacZ $[\beta$-galactosidase $(\beta$-gal)] transgene expression cassette which replaces the Ad E1 region of the Ad genome. It was constructed and grown to high titers on human 293 cells as previously described [18]. Purification consisted of harvesting infected cell lysates, DNase and RNase treatment, and cesium chloride density gradient bandings as per the method of $\mathrm{Ng}$ and Graham [19]. The purified vector preparation was extensively dialyzed against $10 \mathrm{mM}$ Tris $(\mathrm{pH}=8.0)$ and was stored in $1 \%$ sucrose, $\mathrm{PBS}$ at $-80^{\circ} \mathrm{C}$. The vector preparation was determined to be free of replication competent adenovirus by PCR using E1 specific primers and titered by SDS disruption and $\mathrm{OD}_{260}$ spectrophotometry, essentially as previously described $[4,8]$. The titer was further evaluated by in vitro transduction of 293 cells and enumeration of bacterial $\beta$-gal staining cells as previously described. The viral particle (v.p.) bacterial $\beta$-gal transducing unit titer was approximately 13:1, while the v.p. titer:infectious unit titer $\left(\operatorname{TCID}_{50}\right)$ was approximately 120:1 (data not shown) [20,21].

\section{Animal Procedures}

All animal procedures were approved by the Michigan State University animal care and use committee. Adult C57BL/6 mice, B6;129S1-Tlr3 ${ }^{t m 1 F l v} / \mathrm{J}, \mathrm{C} 57 \mathrm{BL} / 10$ and C57BL/10ScNJ (TLR4-KO) were purchased from The Jackson Laboratory (Bar Harbor, Me., USA). MyD88-KO and TRIF-KO mice, on the C57BL/6 background, were kindly provided by Dr. Shizuo Akira. Virus treatment of animals (2-4 months in age) consisted of intravenous injection (via the retro-orbital sinus) of a phosphate-buffered saline solution (PBS, pH 7.4), containing either $1.5 \times 10^{11}$ or $7.5 \times$ $10^{10}$ total virus particles of [E1-]Ad5-LacZ, as previously described [8]. Plasma and tissue samples were obtained and processed at the indicated times post injection as previously described [8]. 
Cytokine and Chemokine Analysis

A uniquely designed multiplex-based assay was used to determine the indicated cytokine/chemokine plasma concentrations, as per the manufacturer's instructions (Bio-Rad, Hercules, Calif., USA) via Luminex 100 technology (Luminex, Austin, Tex., USA), essentially as previously described [8].

\section{Western Blotting}

The Western blotting procedure was completed exactly as previously described [5]. In brief, liver tissue lysates were prepared in lysis buffer (20 mM Tris- $\mathrm{HCl}, \mathrm{pH}$ 7.4, 1 mM EDTA, 150 $\mathrm{mM} \mathrm{NaCl}$ ) containing $1 \%$ Triton $\mathrm{X}-100$ with protease inhibitors. Equivalent concentrations of protein samples were run on $10 \%$ polyacrylamide gels and transferred onto nitrocellulose membranes and probed with antibodies as described previously using fluorescent secondary antibodies. pERK1/2, and IкB $\alpha$ were from Cell Signaling Technology (Danvers, Mass., USA) ERK2 antibody was from Santa Cruz Biotechnology (Santa Cruz, Calif., USA) and antibody against tubulin was from Sigma-Aldrich (St. Louis, Mo., USA). Blots were scanned and bands quantified using Licor's Odyssey scanner [22]. For data analysis, pERK1/2 bands were normalized to ERK 2 and I $\mathrm{B} \alpha$ normalized to tubulin before quantification.

\section{qRT-PCR Analyses}

qRT-PCR analyses were completed as previously described [5]. In brief, RNA was harvested from approximately $100 \mathrm{mg}$ of frozen tissue using TRIzol reagent (Invitrogen, Carlsbad, Calif., USA) as per the manufacturer's protocol. Following RNA isolation, reverse transcription was performed on $180 \mathrm{ng}$ of total RNA using SuperScript II (Invitrogen) reverse transcriptase and random hexamers (Applied Biosystems, Foster City, Calif., USA) as per the manufacturer's protocol. qPCR was carried out on an ABI 7900HT Fast Real-Time PCR system using SYBR Green PCR Mastermix (Applied Biosystems). The comparative Ct method was used to determine relative gene expression using GAPDH to standardize expression levels across all samples. Relative expression increases were calculated based on levels of a respective transcript quantified in mock injected animals of the same genotype. No statistical differences in gene expression were observed in mock injected TLR3-KO, TLR4-KO or TRIF-KO mice compared to their appropriate wild-type controls.

\section{Antibody Titering Assay}

ELISA based titering experiments were completed essentially as previously described [23]. Briefly, $5 \times 10^{8} \mathrm{v}$.p./well or $2 \mu \mathrm{g} \beta$ gal protein/well (each diluted in PBS) were used to coat wells of a 96 -well plate overnight at $4^{\circ} \mathrm{C}$. Plates were washed with $\mathrm{PBS}$ Tween $(0.05 \%)$ solution, and blocking buffer (3\% BSA in PBS) was added to each well and incubated for $1 \mathrm{~h}$ at room temperature. For titering of total IgG antibodies, plasma was diluted 1:800 in blocking buffer, added to the wells and incubated at room temperature for $1 \mathrm{~h}$. Wells were washed using PBS-Tween (0.05\%) and HRPconjugated rabbit anti-mouse antibody (Bio-Rad) was added at a 1:4000 dilution in PBS-Tween. TMB (Sigma-Aldrich) substrate was added to each well, and the reaction was stopped with $2 \mathrm{~N}$ sulfuric acid. Plates were read at $450 \mathrm{~nm}$ in a microplate spectrophotometer. Subisotype titering was completed using hybridoma subisotyping kit (Calbiochem, La Jolla, Calif., USA) using plasma at a dilution of 1:200.

\section{Platelet Enumerations}

Measures of thrombocytopenia were made exactly as previously described [2]. In brief, using the Unopette system (Fisher Scientific, Waltham, Mass., USA), RBCs were lysed and platelets were counted using a Neubauer hemocytometer.

\section{Results}

The Role of TRIF, and TRIF-Utilizing Receptors, TLR3 and TLR4, in TLR Signaling Pathways following Ad Vector Injection

Subsequent to Ad-mediated gene transfer in vivo, we first analyzed the role of TRIF in Ad-induced IкB $\alpha$ degradation (a reliable indicator of $\mathrm{NF \kappa B}$ activation), as well as the rapid induction of ERK1/2 phosphorylation in liver homogenates. To accomplish this, we intravenously injected $1.5 \times 10^{11}$ v.p. of Ad5-LacZ into wild-type and TRIF-KO (both on a C57BL/6 background) mice and assessed the activation kinetics of these pathways at 0,15 , 30, 45 and 60 min post injection (m.p.i.). We confirmed that a significant degradation of the Iк $\mathrm{B} \alpha$ protein and upregulation of phospho-ERK1/2 levels at 15 m.p.i occurred in Ad-injected wild-type animals, both of which returned to baseline levels by 60 m.p.i (fig. 1a, b) [5]. In contrast, IкB $\alpha$ levels had already returned to basal levels by 45 m.p.i in Ad-injected TRIF-KO mice ( $<<0.01)$, indicating a significant role for TRIF in maintaining the activation of NFKB subsequent to Ad-mediated gene transfer. In addition, we also observed a significant delay in the induction of phospho-ERK1/2 in Ad-treated TRIF$\mathrm{KO}$ mice, a response which peaked at 30 m.p.i, as compared to identically injected C57BL/6 mice which reached peak phospho-ERK1/2 activation by 15 m.p.i.

Fig. 1. Role of TRIF, TLR3 and TLR4 in Ad-vector induced IкB $\alpha$ degradation and ERK phosphorylation. Wild-type C57BL/6, TRIF-KO (a, b), TLR3-KO (c, d), TLR4-KO (e, f) mice were injected intravenously with adenoviral vectors $\left(1.5 \times 10^{11}\right.$ viral particles per mouse). The livers were collected at the indicated time points, snap frozen and lysates extracted as described in 'Materials and Methods'. IкB $\alpha$ and tubulin (a, c, e) and p-ERK1/2 and ERK2 (b, d, f) levels were determined by Western blot analysis using Licor's Odyssey. Quantification was performed after normalizing the $\mathrm{p}$-ERK levels to ERK2 and IкB $\alpha$ levels to Tubulin, to control for loading. $\mathrm{n}=3$ for all groups of mice. For each analysis, quantification is shown on the top and a representative blot on the bottom. The bars represent mean \pm SD. Statistics were completed using 1-way ANOVA followed by Tukey's post-hoc test. ${ }^{*} \mathrm{p}<0.05$; ${ }^{* *} \mathrm{p}<0.01 ;{ }^{* *} \mathrm{p}<0.001$. n.s. $=$ not significant; $\mathrm{NI}=$ no injection. 

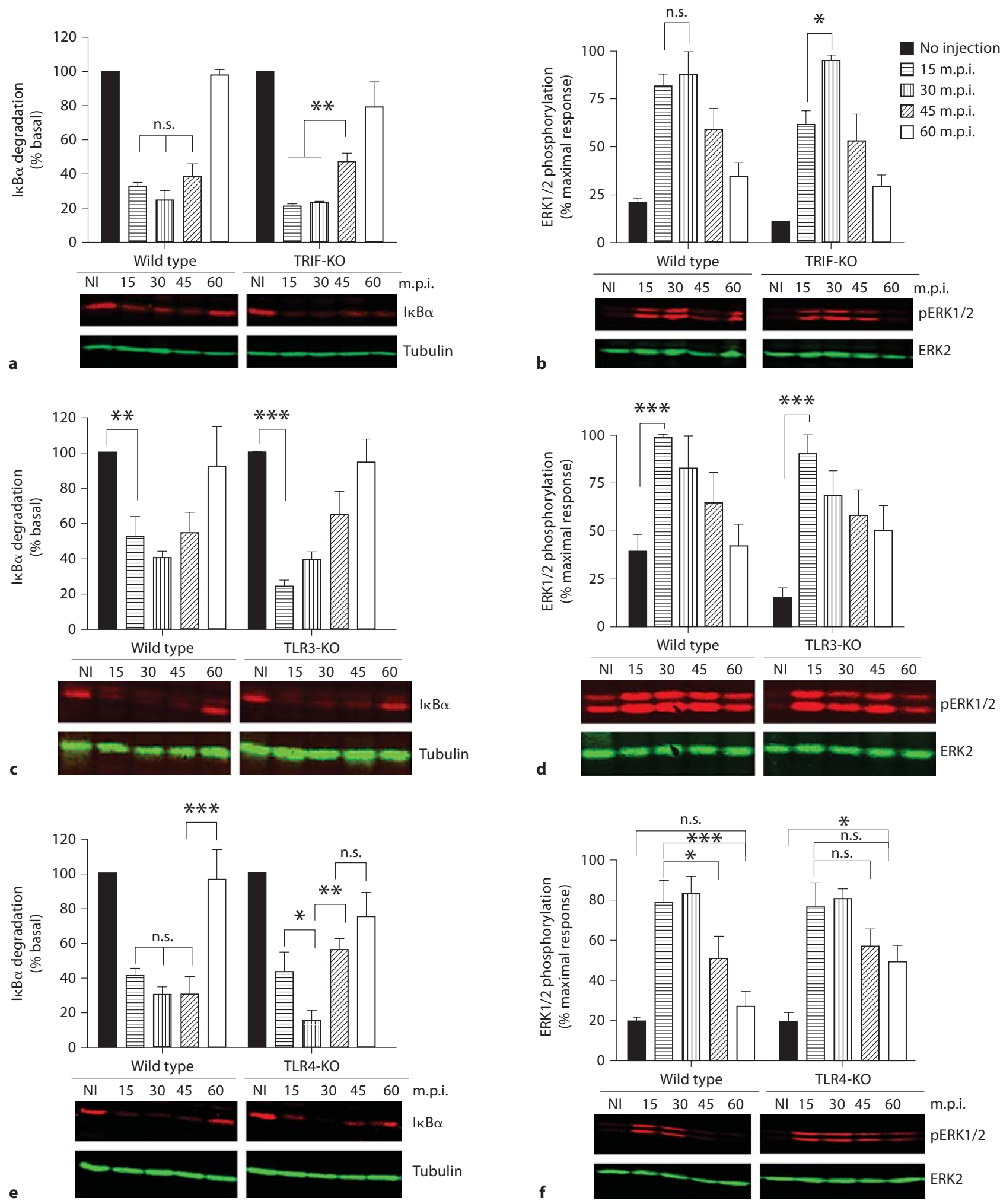
Table 1. Ad-induced gene expression in liver (fold increase over mock, 6 h.p.i.)

\begin{tabular}{|c|c|c|c|c|c|}
\hline & C57BL/6 & TLR3-KO & TRIF-KO & C57BL10 & TLR4-KO \\
\hline IRF-7 & $10.3 \pm 2.5$ & $14 \pm 1.9$ & $13.9 \pm 2.3$ & $14.9 \pm 1.7$ & $7.27 \pm 2.08$ \\
\hline IRF-8 & $1.9 \pm 0.1$ & $2.2 \pm 0.49$ & $2.8 \pm 0.7$ & $2.67 \pm 0.97$ & $1.9 \pm 0.29$ \\
\hline MyD88 & $5.95 \pm 1.6$ & $3.78 \pm 0.7$ & $3.67 \pm 0.79$ & $4.71 \pm 0.08$ & $4.72 \pm 0.72$ \\
\hline NOD1 & $2.1 \pm 0.59$ & $2.08 \pm 0.3$ & $1.9 \pm 0.5^{\mathrm{a}}$ & $2.9 \pm 0.7$ & $2.0 \pm 0.4$ \\
\hline SOCS1 & $102.5 \pm 27.5$ & $51.6 \pm 18.2$ & $22.4 \pm 9.8$ & $90.5 \pm 24.0$ & $45.3 \pm 8.44$ \\
\hline SOCS3 & $3.9 \pm 0.5$ & $3.1 \pm 0.45$ & $3.9 \pm 1.03$ & $9.8 \pm 2.0$ & $7.0 \pm 2.2$ \\
\hline TBK1 & $4.7 \pm 1.3$ & $3.6 \pm 0.4$ & $3.9 \pm 0.5$ & $4.2 \pm 1.1$ & $3.5 \pm 0.9$ \\
\hline TLR2 & $61.0 \pm 21.1$ & $23.7 \pm 6.9$ & $20.6 \pm 11.5$ & $72.3 \pm 18.2$ & $55.2 \pm 24.5$ \\
\hline TLR3 & $28.0 \pm 12.7$ & $17.3 \pm 5.3$ & $10.5 \pm 2.3^{\mathrm{a}}$ & $21.1 \pm 4.1$ & $13.8 \pm 4.8$ \\
\hline TLR9 & $3.7 \pm 0.8$ & $6.3 \pm 1.2$ & $4.4 \pm 3.7$ & $6.5 \pm 2.2$ & $3.8 \pm 0.8$ \\
\hline TRAF2-bp & $9.8 \pm 7.4^{\mathrm{a}}$ & $10.8 \pm 2.6$ & $8.8 \pm 3.5$ & $18.2 \pm 4.8$ & $16.8 \pm 5.5$ \\
\hline
\end{tabular}

The numbers represent mean \pm SD. Statistical analysis was completed using 1-way ANOVA with a StudentNewman-Keuls post-hoc test. All results apart from those marked with a were significantly different $(\mathrm{p}<0.05)$ vs. mock; results shown in bold were significantly different $(\mathrm{p}<0.05)$ vs. C57BL/6 mice injected with virus. Values obtained from TLR4-KO mice are compared to values obtained from C57BL10 control animals using a homoscedastic t test.

Confirming a significant role for TRIF in the kinetics of both Iк $B \alpha$ degradation and phospho-ERK1/2 upregulation subsequent to Ad vector-mediated transduction in vivo, we also evaluated the role of TLR3 and TLR4 in these responses, as these 2 TLRs are known to utilize TRIF as an adaptor protein in innate immune response signaling [24]. Although we did not see significantly different patterns of IкB $\alpha$ degradation or phospho-ERK1/2 induction in liver homogenates derived from Ad-injected, TLR3-deficient animals (fig. 1c, d), we observed dramatically altered kinetics of $\mathrm{I} \kappa \mathrm{B} \alpha$ degradation and ERK1/2 phosphorylation in Ad-injected TLR4-KO mice (fig. 1e, f). Specifically, we observed significantly more Iк $\mathrm{B} \alpha$ degradation at 30 m.p.i compared to both 15 and 45 m.p.i ( $\mathrm{p}<0.05)$ in Ad-injected TRL4-KO mice relative to identically injected wild-type mice, and in stark contrast to our results utilizing TRIF-KO mice. Despite this significant elevation in the NFKB activation pathway at 30 m.p.i, we again observed that I $\mathrm{B} \alpha$ levels rapidly returned to basal levels by 45 m.p.i, $(\mathrm{p}<0.001)$ paralleling the previous observations we made in TRIF-deficient animals, indicating that some aspects of the TRIF-dependent Adinduced innate immune response are mediated by Ad interactions with TLR4. We also observed prolonged phosphorylation of ERK1/2 subsequent to Ad injection into TLR4-KO mice, which continued past the 60 m.p.i time point tested in this analysis $(\mathrm{p}<0.05)$. In accord with increased degradation of $\mathrm{I} \kappa \mathrm{B} \alpha$ at 30 m.p.i, these latter re- sults suggest that TLR4 acts as a negative regulator of Adinduced innate immune signaling in murine liver tissues.

The Role of TRIF, and TRIF-Dependent TLRs, in Ad Induction of Host Immune Response Genes

We have previously shown that the expression of many innate immune response genes, including those that participate in interferon response networks (IRFs, TBK1), TLR signaling molecules (TLRs, TRAF2-bp, MyD88), other pathogen-recognition receptor proteins (NOD1) and negative regulators of cytokine signaling (SOCS) proteins are dysregulated within $6 \mathrm{~h}$ post injection (h.p.i.) with Ad vectors $[4,7,8]$. To discern the role that TRIF may play in these important responses, we analyzed the expression of a subset of Ad-dysregulated genes in the liver of Ad-injected TRIF-KO, TLR3-KO and TLR4-KO mice (table 1). Our results confirmed that TLR3, TLR4 and TRIF are each required for the full induction of SOCS-1 transcript subsequent to Ad transduction in vivo $(p<0.05)$. Furthermore, we observed a significant role for TLR3 and TRIF in the induction of TLR2, and a similar requirement for TLR4 in the induction of IRF-7 transcript $(\mathrm{p}<0.05)$. 


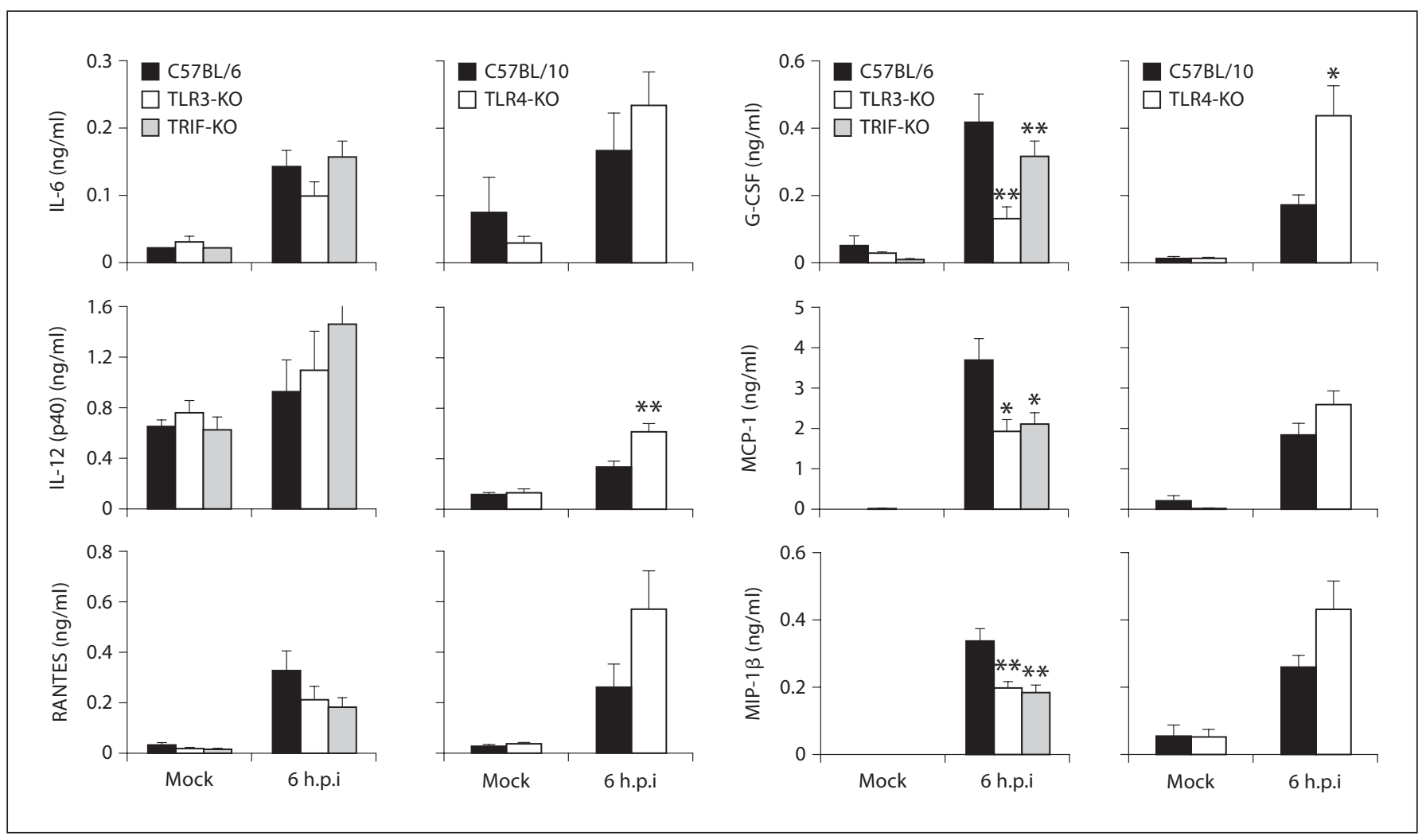

Fig. 2. Dual roles for TRIF utilizing receptors in Ad stimulated induction of cytokines and chemokines. C57BL/6 $(\mathrm{n}=18)$, TLR3KO ( $\mathrm{n}=13)$, TRIF-KO $(\mathrm{n}=18), \mathrm{C} 57 \mathrm{BL} / 10(\mathrm{n}=8)$ and TLR4-KO $(\mathrm{n}=8)$ mice were intravenously injected with $7.5 \times 10^{10}$ v.p./ mouse of Ad5-LacZ or mock injected with PBS ( $\mathrm{n}=3$ for all). Plasma samples were isolated at 6 h.p.i. and analyzed using a custom multiplex bead assay from Bio-Rad. The bars represent the mean \pm SE. Statistical analyses consisted of ANOVA followed by Newman-Keuls post-hoc test for C57BL/6, TLR3-KO and TRIF$\mathrm{KO}$ comparisons. A homoscedastic 2-tailed t test was used to compare levels obtained in C57BL/10 mice compared to TLR4$\mathrm{KO}$ animals. ${ }^{*} \mathrm{p}<0.05 ;{ }^{* *} \mathrm{p}<0.01$, vs. identically injected wildtype mice.

\section{Dual Roles for TRIF-Dependent TLRs in Systemic Cytokine Responses following Intravenous Ad Injection}

Activation of both NFKB and MAPK pathways results in several innate immune responses, including induction of the cytokine and chemokine storms that can follow pathogenic exposures. We and others have previously identified a positive role for TLR2, TLR9, and MyD88 in the systemic influx of many important cytokines and chemokines noted shortly after systemic injection of Adbased vectors into mice $[5,6,11,12]$. Some of these same inflammatory mediators have been noted to be induced in human clinical trials utilizing Ad-based vectors, justifying these animal models as clinically relevant [25]. Based upon these considerations, we evaluated the roles of TRIF, and the TRIF-utilizing TLR3 and TLR4 recep- tors, in the induction of 6 cyto-/chemokines known to be induced following injection of Ad-based vectors. At 6 h.p.i. of $7.5 \times 10^{10}$ v.p., we observed a significant role for TLR3 in the Ad-mediated induction of G-CSF $(p<0.01)$, and significant roles for both TLR3 and TRIF in similar inductions of MCP-1 ( $\mathrm{p}<0.05)$ and MIP-1 $\beta(\mathrm{p}<0.01)$ (fig. 2). Interestingly, we observed a consistent trend that the cytokine responses quantified in TLR4-KO animals were higher than those induced in identically injected, wild-type mice. However, only the induction of IL$12(\mathrm{p} 40)(\mathrm{p}<0.01)$ and G-CSF $(\mathrm{p}<0.05)$ reached statistical significance. Unlike our previous studies confirming MyD88's importance subsequent to Ad-mediated gene transfer, we did not observe a significant role for TRIF, TLR3 or TLR4 in the induction of IL- 6 or RANTES at 6 h.p.i.. Additionally, Ad induction of thrombocytopenia 
was not affected by TLR3, TLR4, or TRIF deficiencies at 24 h.p.i., suggesting that Ad-induced thrombocytopenia is independent of TRIF and TRIF-dependent TLRs (data not shown). Furthermore, lack of TRIF or TRIF-dependent TLRs did not alter the ability of the Ad vector to transduce liver tissues, as levels of Ad vector-derived gene expression and Ad genome copy numbers measured in the livers of all Ad treated mice were not affected at the 24 h.p.i. time point (data not shown).

\section{Robust Induction of Humoral Immune Responses} to an Ad Vector-Expressed Transgene Is Partially Mediated by TRIF, TLR3, and TLR4

We have previously shown that subsequent to Ad-vector injection, adaptive immune responses generated against the Ad vector-expressed transgene, both cellular and humoral, require MyD88-mediated receptor signaling [8]. Similarly, we have shown that TLR2 is partially required for mediating humoral responses to an Ad vector-expressed transgene and generation of anti-Ad neutralizing antibodies following Ad vector administration [5]. Since deficiency in MyD88 did not completely abrogate these responses, we hypothesized that TRIF may also modulate some of these adaptive immune responses, potentially by mediating TLR 3 and/or TLR4 signaling triggered by Ad vectors. We addressed this possibility by quantifying transgene (LacZ; also referred to as $\beta$-galactosidase) and Ad-specific antibody responses at weekly intervals following systemic injection of $7.5 \times 10^{10}$ v.p. of an Ad vector expressing $\beta$-gal into normal, TRIF, TLR3- or TLR4-KO mice. Our results indicate that the lack of TRIF signaling results in a slight, yet statistically significant increase in total IgG levels directed against $\beta$-gal subsequent to treatment with Ad5-LacZ (fig. 3). Furthermore, we observed significantly higher levels of $\beta$-gal-specific IgG2c, IgG2b and IgG3 levels $(p<0.05)$ in TRIF-deficient mice, the latter paralleled results obtained in TLR3-deficient mice, suggesting a TLR3/TRIF-mediated suppression of this important antibody subisotype subsequent to Ad vector treatment $(\mathrm{p}<0.05)$.

We hypothesized that a TLR4/TRIF-mediated pathway may account for the observed increases in levels of anti- $\beta$-gal IgG2c and IgG2b observed in Ad-injected TRIF-KO mice, since we found increased proinflammatory cytokine responses in Ad-treated TLR4-KO mice. However, we did not observe a significant role for TLR4 in the induction of these 2 antibody subisotypes. Interestingly, the level of $\beta$-gal-specific total IgG in TLR4-KO animals was slightly decreased 4 weeks post injection compared to identically injected C57BL/10 control mice, and $\beta$-gal-specific IgG1 and IgG3 levels were significantly lower at several time points post Ad injection.

The induction of Ad capsid-specific humoral responses was also partially mediated by TRIF, TLR3, and TLR4. Specifically, we observed significantly higher levels of Ad-specific total IgG and IgG2c 4 weeks post injection in Ad-treated TRIF-KO animals as compared to identically injected C57BL/ 6 control mice $(\mathrm{p}<0.05)$. While complex, these results suggest that inductions of proinflammatory cytokine early after Ad treatment may not positively correlate with surrogate markers of downstream adaptive immune responses, a result we have noted in previous studies utilizing alternative serotype Ad-based vectors [26]. We also observed significantly lower levels of antiAd-specific IgG1 levels at $14(\mathrm{p}<0.01)$ and $21(\mathrm{p}<0.05)$ days after injection (d.p.i.) of the Ad5-LacZ vector into TRIF-KO mice relative to similarly treated wild-type mice. This result indicates a significantly different role for TRIF in mediating the anti-transgene versus the antiAd capsid humoral immune responses generated subsequent to Ad vector administration.

Relative to the role of TRIF-dependent TLRs and the induction of antibody responses to Ad capsids, our results demonstrated that TLR3 partially mediates anti-Ad-specific IgG1, IgG2c and IgG3 antibody isotype responses. Furthermore, we found that TLR4 is also required for the complete IgG3 response at 14 d.p.i. ( $<<0.01)$, and that TLR4 plays an inhibitory role in the anti-Ad-specific IgG2c responses noted at 14 d.p.i. $(\mathrm{p}<0.01)$. We did not identify a role for TRIF, TLR3, or TLR4 in the generation of Ad-neutralizing antibody titers at any time point tested (data not shown). Together, these studies suggest the existence of a complex mechanism mediating induction of robust humoral immune responses to Ad vectors, and/or the transgene-derived proteins they express, one that potentially requires complicated interactions with TLR3- and TLR4-mediated signaling pathways.

\section{Additive Role for TRIF and MyD88 in Innate \\ Toxicities Associated with Systemic Ad Vector \\ Administration}

Coupled with previous studies, these results establish a clear role for both MyD88 and TRIF, and the receptors that require them, in both innate and adaptive immune responses to Ad-based vectors [7, 8]. However, the importance of each of these important adaptor proteins, relative to each other in these responses, has not been elucidated to date. For this reason, we derived MyD88 and TRIF double knockout animals (MyD88 ${ }^{-/-} \mathrm{TRIF}^{-/-} \mathrm{Dbl}-\mathrm{KO}$ ) to investigate this question. 

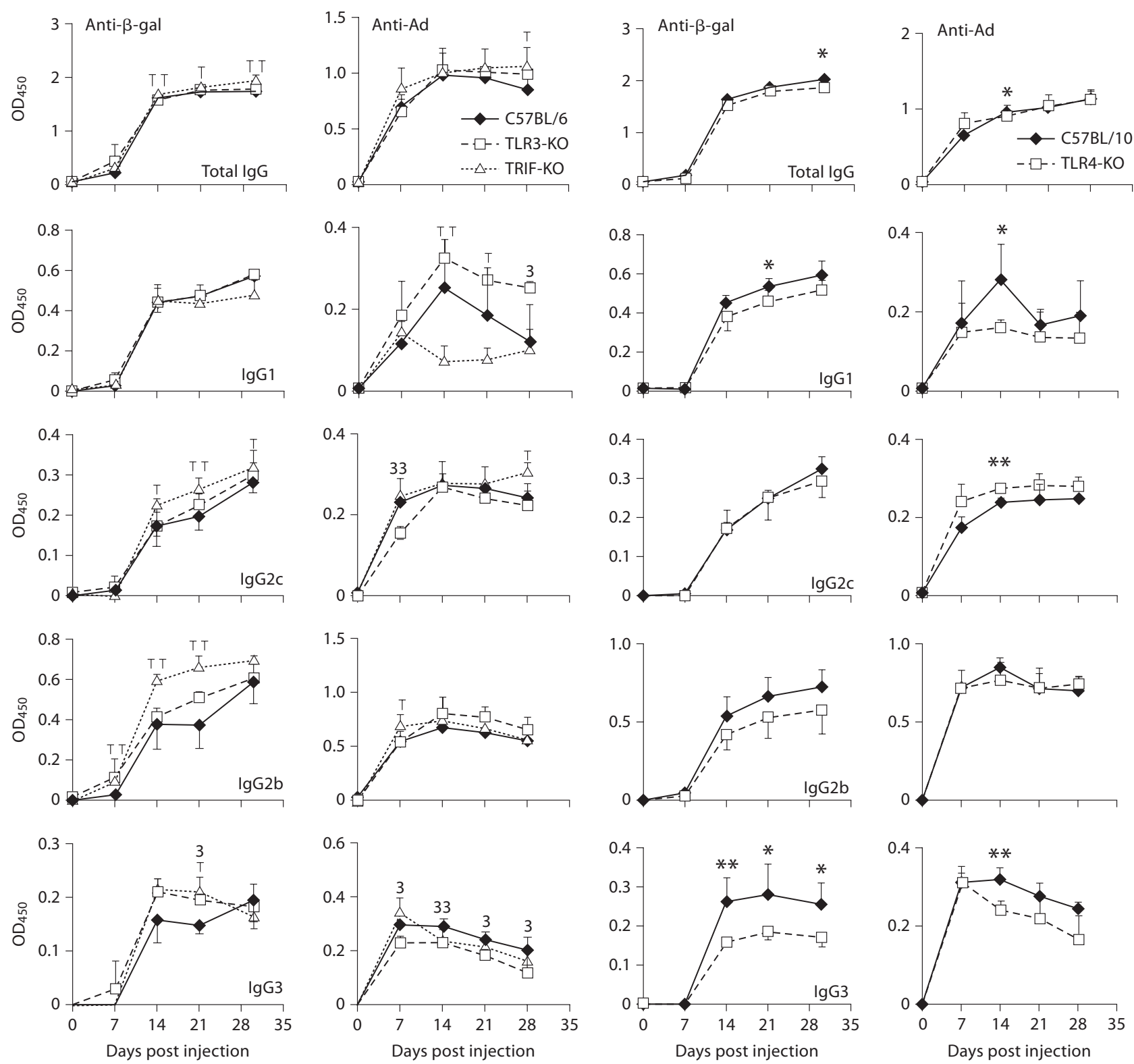

Fig. 3. Partial roles for TRIF-utilizing TLRs in antibody responses generated against Ad vectors and their encoded transgene ( $\beta$ galactosidase). C57BL/6 $(\mathrm{n}=5)$, TLR3-KO $(\mathrm{n}=4)$, TRIF-KO $(\mathrm{n}=$ $5), \mathrm{C} 57 \mathrm{BL} / 10(\mathrm{n}=3)$ and TLR4-KO $(\mathrm{n}=5)$ were intravenously injected with $7.5 \times 10^{10}$ v.p./mouse of Ad5-LacZ. Anti- $\beta$-gal and anti-Ad-specific antibody titers were evaluated at the appropriate plasma dilutions. Bars represent mean \pm SD. A homoscedastic 2-tailed t test was used to compare levels obtained in knockout animals with those in control wild-type animals at each time point. T $=$ TRIF-KO; $3=$ TLR3-KO. A single $(\mathrm{p}<0.05)$ or double $(\mathrm{p}<0.01)$ character indicates statistically different values compared to identically injected wild-type mice. ${ }^{*} \mathrm{p}<0.05$; ${ }^{* *} \mathrm{p}<$ 0.01 , TLR4-KO vs. C57BL/10 wild-type mice. 
Fig. 4. Ad-induced innate toxicities are partially mediated by both MyD88 and TRIF. C57BL/6 $(\mathrm{n}=5), \mathrm{MyD} 88-\mathrm{KO}(\mathrm{n}=$ 4), TRIF-KO $(\mathrm{n}=5)$, and DBL-KO $(\mathrm{n}=4)$ were intravenously injected with $7.5 \times$ $10^{10}$ v.p./mouse of Ad5-LacZ. a Thrombocytopenia was evaluated at 24 h.p.i. by enumeration of blood platelet levels. Bars represent mean \pm SD. $\mathbf{b}$ Plasma levels of cytokines and chemokines were measured at 6 h.p.i. using a custom multiplex bead assay from Bio-Rad. Bars represent the mean \pm SE. For all comparisons, a homoscedastic 2-tailed t test was used to compare levels obtained in knockout animals compared to control wild-type animals. The dashed line indicates values obtained in TRIF-KO animals. ${ }^{*} \mathrm{p}<0.05$; ${ }^{* *} \mathrm{p}<0.01$, vs. identically injected wildtype mice.
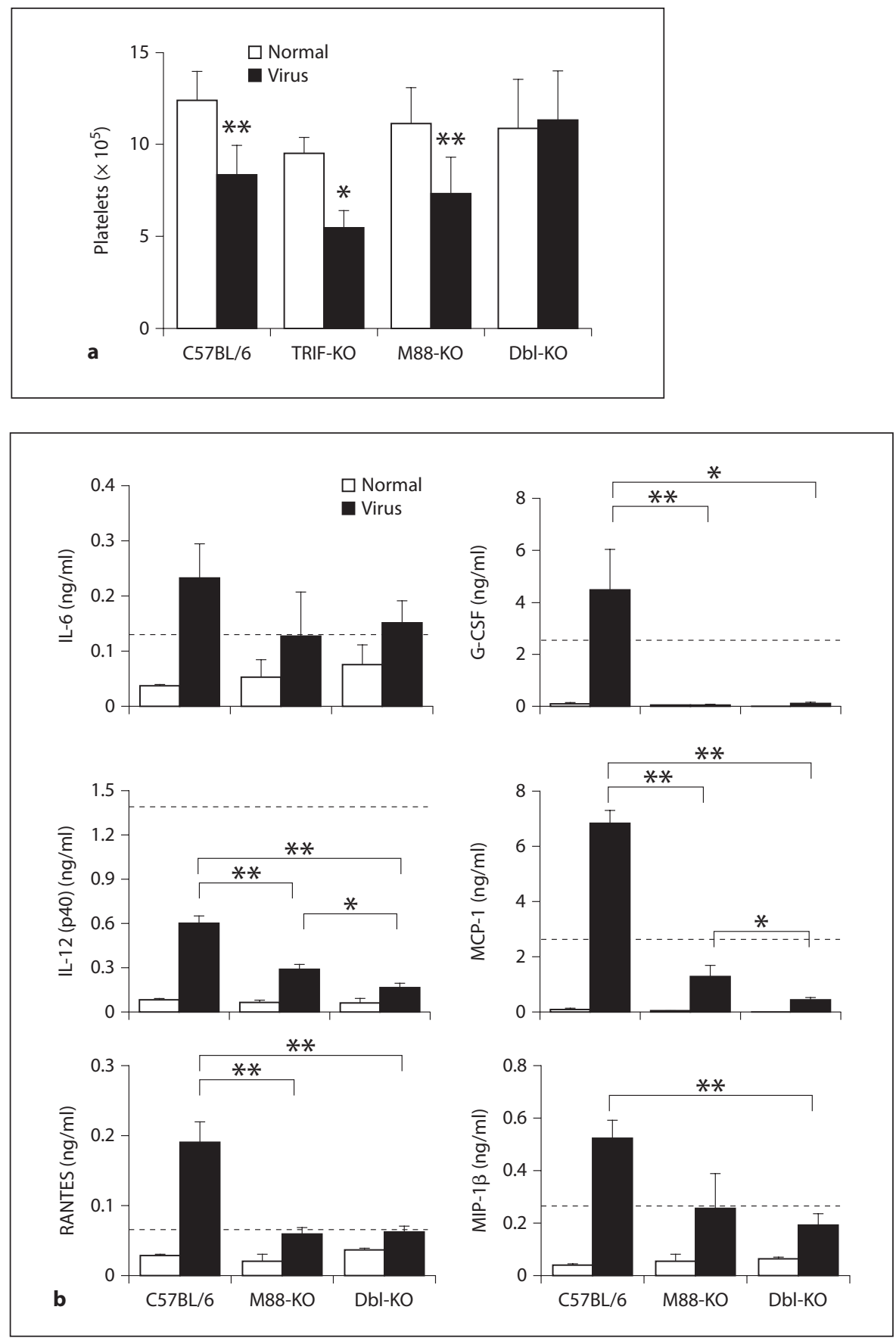

Typically regarded as a consumptive process, acute thrombocytopenia is observed after naturally acquired Ad infections and shortly after systemic injection of Adbased vectors [27-29]. We have previously shown that this process requires a functional complement system, and is specifically mediated by the alternative complement pathway $[2,4]$. However, we have not observed an individual role for TLR2, TLR4, TLR3, TRIF, or MyD88 in this response, as mice deficient in any single protein do not ameliorate this process (unpublished observations and [8]). Surprisingly, MyD88 ${ }^{-/-} \mathrm{TRIF}^{-/-}$Dbl-KO mice are not susceptible to the acute thrombocytopenia induced by systemic administrations of Ad vectors at 24 h.p.i. (fig. 4a). This result suggests that both TLR adaptor proteins may play a role in this important response, and may indirectly suggest a potential for crosstalk between 


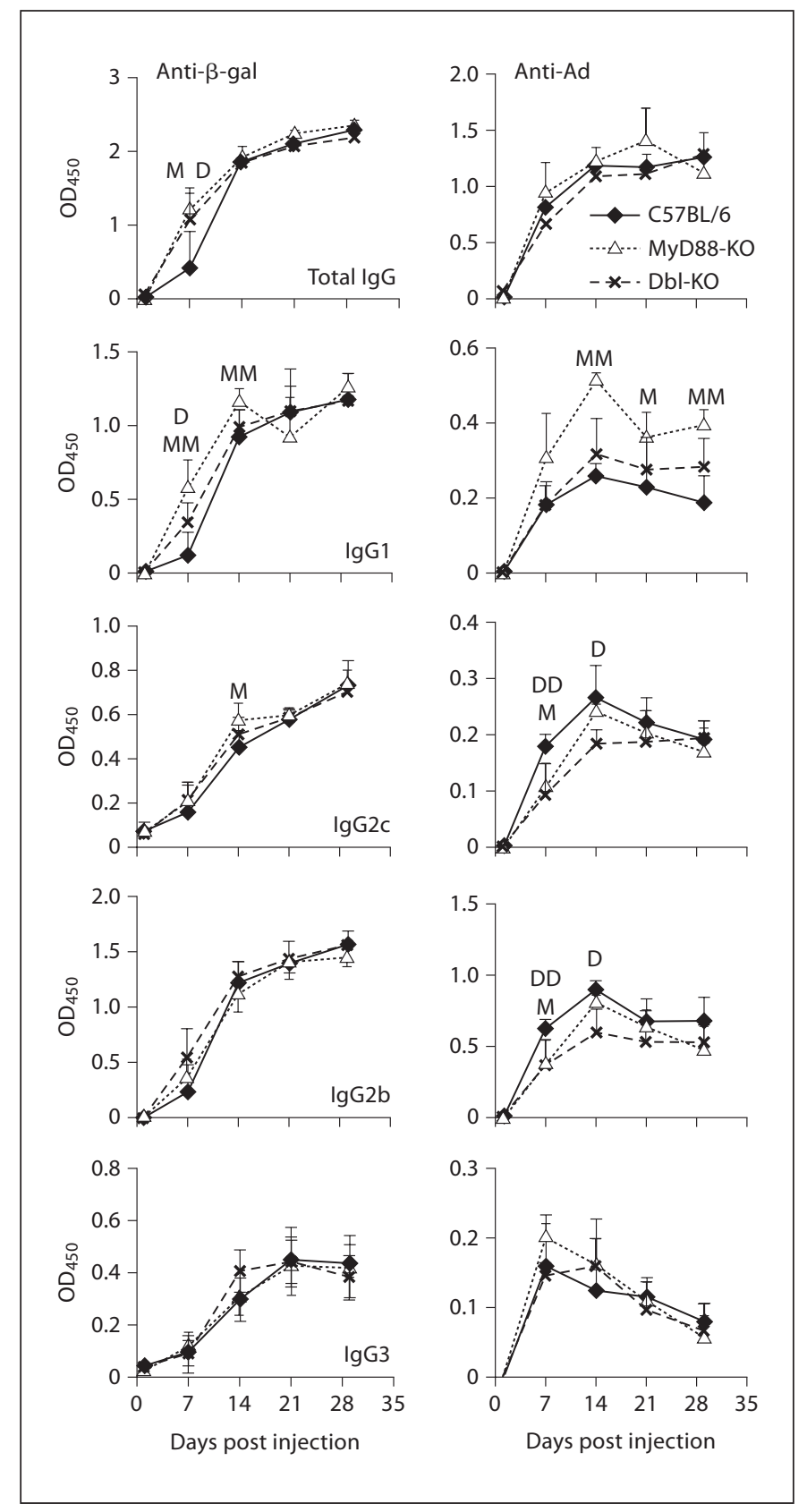

Fig. 5. Both MyD88 and TRIF mediate the Ad- and transgenespecific humoral responses following systemic Ad-vector injection. C57BL/6 $(\mathrm{n}=5), \mathrm{MyD} 88-\mathrm{KO}(\mathrm{n}=4)$ and Dbl-KO $(\mathrm{n}=5)$ were intravenously injected with $7.5 \times 10^{10} \mathrm{v}$.p./mouse of Ad5-LacZ. Anti- $\beta$-gal and anti-Ad-specific antibody titers were evaluated at the appropriate plasma dilutions. Bars represent mean $\pm \mathrm{SD}$. A homoscedastic 2-tailed t test was used to compare levels obtained in knockout animals compared to control wild-type animals at each time point. $\mathrm{M}=\mathrm{MyD} 88-\mathrm{KO} ; \mathrm{D}=\mathrm{TRIF}^{-/-} \mathrm{MyD}^{-/-} \mathrm{Dbl}-\mathrm{KO}$ mice. A single $(\mathrm{p}<0.05)$ or double $(\mathrm{p}<0.01)$ letter indicates statistically different values compared to identically injected wildtype mice. ancient complement pathways and TLR pathways in this response, since we have previously noted that Ad-induced thrombocytopenia is also dependent upon interactions with the alternative pathway of complement activation $[2,4]$.

To determine whether MyD88 or TRIF additively or redundantly regulate other Ad-induced innate immune responses, we measured the release of a select number of cytokines and chemokines at 6 h.p.i. in Ad-injected

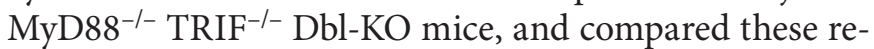
sults to identically treated MyD88-KO mice (fig. 4b). Although our results reconfirm a dominant role for MyD88 in mediating the induction of many Ad-induced inflammatory responses [i.e. Ad vector induction of IL-12(p40), MCP-1, G-CSF, and RANTES] our results also revealed TRIF as being required for the full induction of IL-12(p40), indicating that, together, both MyD88 and TRIF are required for the full induction of this cytokine. Interestingly, we also detected an additive role for both MyD88 and TRIF in the induction of MCP-1 $(\mathrm{p}<0.05)$ by Ad vectors.

We next evaluated the $\beta$-gal and Ad-specific humoral responses generated in Ad5-LacZ-treated MyD88 ${ }^{-/-}$ $\mathrm{TRIF}^{-/-}$Dbl-KO mice as compared to identically treated wild-type mice, or mice singly deficient in MyD88 (fig. 5). In addition to the inhibitory role for TRIF, we also observed inhibitory roles for MyD88 during the induction of $\beta$-gal-specific total IgG $(p<0.05)$ and IgG1 $(p<0.01)$ subisotype antibodies at early time points. Interestingly, many of the $\beta$-gal-specific antibody responses quantified in individual knockout animals returned to normal levels in mice deficient in both MyD88 and TRIF, an intriguing result we elaborate upon in the 'Discussion'.

In our examination of Ad-specific antibody responses, we found that capsid-specific IgG levels were not different between the various strains of Ad-injected mice. In contrast, we noted a significant inhibitory role for MyD88 in the induction of Ad capsid-specific IgG1, as plasma levels of this antibody were significantly higher than those quantified in the plasma of identically treated wildtype mice at 14, 21 and 28 d.p.i. $(\mathrm{p}<0.01, \mathrm{p}<0.05$ and $\mathrm{p}<0.01$, respectively). The induction of Ad capsid-specific IgG1 returned to normal levels in Ad-injected double knockout mice, as these levels were not significantly different to those measured in identically treated wildtype mice. Achievement of maximal titers of anti-Adspecific IgG2c antibody levels also required MyD88 but was noted to be reduced at 14 d.p.i. in Ad-treated double knockout mice relative to identically treated MyD88-KO mice at this same time point. The induction of anti-Ad IgG2b levels followed a similar pattern as that noted for 
anti-Ad IgG2c in the various strains of mice. Interestingly, no significant difference in Ad capsid-specific total IgG, IgG2c, IgG2b or IgG3 plasma antibody levels were observed in any mouse strain 4 weeks post vector injection. Finally, there was not a detectable difference in Ad genome numbers, $\beta$-gal activity or X-gal staining in liver tissues derived from Ad injected TRIF-KO, MyD88-KO or double knockout animals as compared to identically injected wild-type mice at 28 d.p.i. (data not shown).

\section{Discussion}

In light of recent HIV vaccine trials using human Ad5based vectors, it has become increasingly important to evaluate the mechanism by which Ads trigger both innate and adaptive immune responses, as such knowledge may foster more efficacious usage of this important and widely utilized gene transfer platform [30]. It has been well established that innate immune responses impact upon both humoral and cellular adaptive immune responses [31]. It has also been shown that innate immune receptors, specifically TLRs, are expressed not only on cells known for innate immune signaling, but also on cells comprising the adaptive compartment [32]. We have recently shown that 2 important arms of the innate immune system, the complement system and the TLR pathways, play important roles in mediating these responses subsequent to Ad vector treatments $[2,4,5,8]$. Specific to TLR signaling, we have shown that portions of the innate and adaptive immune responses to an Ad vector-expressed transgene are partially mediated by the TLR adaptor MyD88 [8].

TLR9 and IL-1R, 2 other innate immune receptors that require the MyD88 adaptor protein, are required for the full induction of innate immune responses to Adbased vectors [6, 10-12]. More recently, we confirmed that the MyD88-dependent TLR2 is partially required for maximal induction of neutralizing antibodies generated against the virus itself, as well against an Ad vector-expressed transgene [5]. In light of these data, MyD88-mediated signaling can only partially explain the robust innate and adaptive immune responses generated against both the Ad vector itself and the transgene that it expresses. Consequently, we hypothesized that another TLR adaptor pathway may also play a role in these important immune responses noted after Ad vector-mediated gene transfer. Specifically we hypothesized that TRIF and TRIF-dependent TLRs (i.e. TLR3 and TLR4) may also modulate both innate and adaptive immune responses generated against Ad-based vectors and the transgenes they express.

Our previous studies have revealed that the induction of ERK1/2 phosphorylation is independent of MyD88 and unique to liver resident antigen presenting cells [5]. The experiments in this study suggest that TRIF is partially involved in the initiation of this response. We next reasoned that these altered kinetics were a result of the interaction of Ad5-based vectors with TLR3 and/or TLR4. Examination of this possibility in TLR3-deficient mice did not reveal a significant role for this receptor in regulating ERK1/2 phosphorylation. In contrast, examination of Ad-treated TLR4-KO mice revealed altered kinetics of ERK1/2 phosphorylation, however the results suggested that TLR4 is a negative regulator of Ad vector-based innate immune signaling in the murine liver. Adding support to this novel finding, we observed a similar negative role for TLR4 in mediating the degradation of $\mathrm{I \kappa} B \alpha$.

The mechanism by which TLR4 functions as a negative regulator of these responses is currently unclear, but this observation may describe a previously unrecognized role for TLRs. There is clear evidence that TLR4 stimulation results in the activation of both positive and negative regulators of TLR signaling. While positive regulators are obviously important mediators of signaling, negative regulators tend to inhibit signaling so as to curtail or inhibit excessive activation. It is possible that TLR4-mediated negative regulators play a dominant and selective role in Ad-induced signaling, such that in TLR4-deficient mice, the lack of TLR4-mediated activation of dominant inhibitory factors results in excessive Ad-induced signaling and elevations in cytokine release. TLR4 may also exert its inhibitory signals through direct physical interactions with other surface TLRs. In support of this, it has been shown that the TLR homolog RP105 inhibits TLR4 signaling through a direct interaction with the receptor itself [33]. In contrast, the negative role of TLR4 in Adinduced signaling may be indirect, potentially by sequestration of shared adaptor proteins. Our results suggest that future studies investigating these intriguing hypotheses are now warranted.

Our previous analysis of liver transcriptome dysregulation following systemic Ad administrations confirmed that MyD88 plays a major, although partial, role in mediating this Ad-induced response, since the significant dysregulation of a major subset of genes in response to Admediated gene transduction involved in IFN responses remained intact in mice deficient in functional MyD88 protein [8]. It is known that TRIF plays a role in TLR4and TLR3-mediated antiviral responses by upregulating 
IFN response genes [24]. Furthermore, we have previously shown that RNAi-mediated knockdown of TRIF in Ad-infected human cells also results in decreased IFN- $\beta$ and NFKB promoter activation [7]. For these reasons, we analyzed the murine liver transcriptome response in Adtreated TRIF-KO mice as compared to identically treated C57BL/6 mice. Significant genes dysregulated in a TRIFdependent manner included SOCS1 and TLR2, both known to be involved in the anti-adenoviral innate immune response $[5,34]$. Interestingly, these genes were also dysregulated in TLR3-KO mice indicating that although we did not detect a role for TLR3 in the induction of ERK1/2 or NFKB activation, TLR3 does affect the Admediated expression of genes in the liver at this time point subsequent to systemic Ad infusion, likely in an ERK1/2 and/or NFкB independent fashion.

While our results showed evidence that TLR4 acts as a negative regulator of some Ad-induced innate immune responses including activation of $\mathrm{NF \kappa B}$ pathways, and prolongation of ERK1/2 activation, we did not detect a similar role for TLR4 in host gene responses to Ad vectors in this study. However, we did observe a significant role for TLR4 in the induction of both IRF-7 and SOCS1. We also detected significantly higher plasma levels of G-CSF in Ad vector-treated TLR4-deficient mice relative to identically treated control mice. This further supports our conclusion that TLR4 acts as a negative regulator of some Ad-induced immune responses. These contrasting data indicate that the role of TLR4 in anti-Ad immune responses is complicated and cannot be predicted a priori. Future studies will be required to confirm these intriguing observations.

Our results also confirmed roles for TRIF and TLR3 in the Ad-mediated induction of MCP- 1 and MIP- $1 \beta$, results consistent with the known utilization of TRIF by TLR3 subsequent to ligand binding. Utilizing Ad-injected $\mathrm{MyD}^{-/-}$TRIF $^{-/-}$Dbl-KO mice, we revealed that Ad induction of G-CSF was solely dependent upon MyD88, but that full induction of MCP-1 and IL-12(p40) additionally required TRIF functionality. Finally, deficiency in both MyD88 and TRIF did not completely eliminate the capability of Ad vectors to induce RANTES and MIP- $1 \beta$, suggesting that Ad vectors interact with other innate immune response pathways to allow for maximal induction of these 2 cytokines, for example the complement pathway [2, 4].

We have previously shown that the humoral response generated against both the Ad vector itself, as well as the transgene it expresses are, in part, dependent on MyD88 dependent mechanisms, some of which are mediated by TLR2 [5]. However, it is clear that TLR2-mediated re- sponses do not completely explain these adaptive responses, as significant levels of anti-Ad and anti-transgene specific antibodies can be detected in Ad-treated TLR-2 KO mice. We now confirm this, as the results of this study demonstrate that TLR4 can also mediate aspects of the transgene-specific IgG3 response elicited by Ad vectors.

Our results also suggest that TRIF may play a negative role for Ad-mediated induction of various antibody subisotypes including IgG2b, IgG2c, and IgG3, the latter mediated by TLR3. It is, however, unclear how TRIF mediates this effect as our results in TLR3 and TLR4-KO mice indicate that neither of these TLRs is required for Ad vectors to induce maximal antigen-specific IgG2c or IgG2b levels.

Interestingly, continued Ad-expressed transgene and Ad capsid-specific antibody responses were detectable in mice doubly deficient in both TRIF and MyD88, indicating that non-TLR-dependent pathways play active roles in these responses, a result that parallels similar findings in other systems [35]. It has been shown that Ad-induced type I interferon pathways are activated independent of MyD88 or TRIF signaling in vitro, and that Ad-neutralizing antibody production requires type I interferon signaling in vivo $[9,11,17]$. Therefore, it is possible that MyD88/TRIF-independent induction of type I interferons in vivo compensates for the complete loss of TLR-mediated signaling in the generation of Ad-specific humoral responses. Future studies evaluating Ad-induced adaptive immunity in mice deficient in both TLR and type I IFN pathways could clarify the individual roles of these systems in mediating these responses.

This study was designed to elucidate the role of TRIF, and TRIF-utilizing receptors in the generation of both innate and humoral adaptive immune responses generated against both an Ad-based vector and the transgene it expresses. The complex interplay between TRIF and MyD88mediated pathways can sometimes make it difficult to logically predict the individual roles each protein may have in mediating immune responses generated after use of Ad vector-based gene therapy vectors and/or vaccines.

\section{Acknowledgments}

We wish to thank Michigan State University's laboratory animal support facilities for their assistance in the humane care and maintenance of the animals utilized in this work. A.A. was supported by National Institutes of Health grants RO1DK-069884 and P01 CA078673, and also by the Osteopathic Heritage Foundation. 


\section{References}

1 Appledorn DM, Kiang A, McBride A, Jiang $\mathrm{H}$, Seregin S, Scott JM, Stringer R, Kousa Y, Hoban M, Frank MM, Amalfitano A: Wildtype adenoviruses from groups A-F evoke unique innate immune responses, of which HAd 3 and SAd 23 are partially complement dependent. Gene Ther 2008;15:885-901.

-2 Appledorn DM, McBride A, Seregin S, Scott JM, Schuldt N, Kiang A, Godbehere S, Amalfitano A: Complex interactions with several arms of the complement system dictate innate and humoral immunity to adenoviral vectors. Gene Ther 2008;15:1606-1617.

-3 Jiang H, Wang Z, Serra D, Frank MM, Amalfitano A: Recombinant adenovirus vectors activate the alternative complement pathway, leading to the binding of human complement protein $\mathrm{c} 3$ independent of anti-ad antibodies. Mol Ther 2004;10:1140-1142.

-4 Kiang A, Hartman ZC, Everett RS, Serra D, Jiang H, Frank MM, Amalfitano A: Multiple innate inflammatory responses induced after systemic adenovirus vector delivery depend on a functional complement system. Mol Ther 2006;14:588-598.

-5 Appledorn DM, Patial S, McBride A, Godbehere S, Van Rooijen N, Parameswaran N, Amalfitano A: Adenovirus vector-induced innate inflammatory mediators, MAPK signaling, as well as adaptive immune responses are dependent upon both TLR2 and TLR9 in vivo. J Immunol 2008;181:2134-2144.

-6 Cerullo V, Seiler MP, Mane V, Brunetti-Pierri N, Clarke C, Bertin TK, Rodgers JR, Lee B: Toll-like receptor 9 triggers an innate immune response to helper-dependent adenoviral vectors. Mol Ther 2007;15:378-385.

$\checkmark 7$ Hartman ZC, Black EP, Amalfitano A: Adenoviral infection induces a multi-faceted innate cellular immune response that is mediated by the toll-like receptor pathway in a549 cells. Virology 2007;358:357-372.

-8 Hartman ZC, Kiang A, Everett RS, Serra D, Yang XY, Clay TM, Amalfitano A: Adenovirus infection triggers a rapid, MyD88-regulated transcriptome response critical to acute-phase and adaptive immune responses in vivo. J Virol 2007;81:1796-1812.

$\checkmark$ Nociari M, Ocheretina O, Schoggins JW, Falck-Pedersen E: Sensing infection by adenovirus: toll-like receptor-independent viral DNA recognition signals activation of the interferon regulatory factor 3 master regulator. J Virol 2007;81:4145-4157.

10 Shayakhmetov DM, Li ZY, Ni S, Lieber A: Interference with the IL-1-signaling pathway improves the toxicity profile of systemically applied adenovirus vectors. J Immunol 2005; 174:7310-7319.

11 Zhu J, Huang X, Yang Y: Innate immune response to adenoviral vectors is mediated by both toll-like receptor-dependent and -independent pathways. J Virol 2007;81:31703180 .
12 Yamaguchi T, Kawabata K, Koizumi N, Sakurai F, Nakashima K, Sakurai H, Sasaki T, Okada N, Yamanishi K, Mizuguchi H: Role of MyD88 and TLR9 in the innate immune response elicited by serotype 5 adenoviral vectors. Hum Gene Ther 2007;18:753-762.

13 Kawai T, Akira S: TLR signaling. Semin Immunol 2007;19:24-32.

14 Uematsu S, Akira S: Toll-like receptors and type I interferons. J Biol Chem 2007;282: 15319-15323.

15 Hoebe K, Du X, Georgel P, Janssen E, Tabeta K, Kim SO, Goode J, Lin P, Mann N, Mudd S, Crozat K, Sovath S, Han J, Beutler B: Identification of LPS2 as a key transducer of MyD88-independent TIR signalling. Nature 2003;424:743-748.

-16 Yamamoto M, Sato S, Hemmi H, Hoshino K, Kaisho T, Sanjo H, Takeuchi O, Sugiyama M, Okabe M, Takeda K, Akira S: Role of adaptor TRIF in the MyD88-independent toll-like receptor signaling pathway. Science 2003; 301:640-643.

17 Zhu J, Huang X, Yang Y: Type I IFN signaling on both B and CD4 T cells is required for protective antibody response to adenovirus. J Immunol 2007;178:3505-3510.

18 Hodges BL, Evans HK, Everett RS, Ding EY, Serra D, Amalfitano A: Adenovirus vectors with the 100k gene deleted and their potential for multiple gene therapy applications. J Virol 2001;75:5913-5920.

19 Ng P, Graham FL: Construction of first-generation adenoviral vectors. Methods $\mathrm{Mol}$ Med 2002;69:389-414.

20 Amalfitano A, Hauser MA, Hu H, Serra D, Begy CR, Chamberlain JS: Production and characterization of improved adenovirus vectors with the E1, E2b, and E3 genes deleted. J Virol 1998;72:926-933.

- 21 Everett RS, Hodges BL, Ding EY, Xu F, Serra D, Amalfitano A: Liver toxicities typically induced by first-generation adenoviral vectors can be reduced by use of E1, E2b-deleted adenoviral vectors. Hum Gene Ther 2003; 14 : 1715-1726.

22 Parameswaran N, Pao CS, Leonhard KS, Kang DS, Kratz M, Ley SC, Benovic JL: Arrestin-2 and $G$ protein-coupled receptor kinase 5 interact with NFkappaB1 p105 and negatively regulate lipopolysaccharide-stimulated ERK1/2 activation in macrophages. J Biol Chem 2006;281:34159-34170.

-23 Hensley SE, Cun AS, Giles-Davis W, Li Y, Xiang Z, Lasaro MO, Williams BR, Silverman RH, Ertl HC: Type I interferon inhibits antibody responses induced by a chimpanzee adenovirus vector. Mol Ther 2007;15: 393-403.

24 Lee MS, Kim YJ: Signaling pathways downstream of pattern-recognition receptors and their cross talk. Ann Rev Biochem 2007;76: 447-480.
-25 Raper SE, Chirmule N, Lee FS, Wivel NA, Bagg A, Gao GP, Wilson JM, Batshaw ML: Fatal systemic inflammatory response syndrome in a ornithine transcarbamylase deficient patient following adenoviral gene transfer. Mol Genet Metab 2003;80:148158.

26 Hartman ZC, Appledorn DM, Serra D, Glass O, Mendelson TB, Clay TM, Amalfitano A: Replication-attenuated human adenoviral type 4 vectors elicit capsid dependent enhanced innate immune responses that are partially dependent upon interactions with the complement system. Virology 2008;374: 453-467.

27 Eggerman TL, Mondoro TH, Lozier JN, Vostal JG: Adenoviral vectors do not induce, inhibit, or potentiate human platelet aggregation. Hum Gene Ther 2002;13:125-128.

28 Othman M, Labelle A, Mazzetti I, Elbatarny HS, Lillicrap D: Adenovirus-induced thrombocytopenia: the role of von Willebrand factor and P-selectin in mediating accelerated platelet clearance. Blood 2007;109:28322839.

29 Wolins N, Lozier J, Eggerman TL, Jones E, Aguilar-Cordova E, Vostal JG: Intravenous administration of replication-incompetent adenovirus to rhesus monkeys induces thrombocytopenia by increasing in vivo platelet clearance. Br J Haematol 2003;123: 903-905.

30 Cox KS, Clair JH, Prokop MT, Sykes KJ, Dubey SA, Shiver JW, Robertson MN, Casimiro DR: DNA gag/adenovirus type 5 (Ad5) gag and Ad5 gag/Ad5 gag vaccines induce distinct T-cell response profiles. J Virol 2008; 82:8161-8171.

31 van Duin D, Medzhitov R, Shaw AC: Triggering TLR signaling in vaccination. Trend Immunol 2006;27:49-55.

32 Imanishi T, Hara H, Suzuki S, Suzuki N, Akira S, Saito T: Cutting edge: TLR2 directly triggers Th1 effector functions. J Immunol 2007;178:6715-6719.

- 33 Divanovic S, Trompette A, Atabani SF, Madan R, Golenbock DT, Visintin A, Finberg RW, Tarakhovsky A, Vogel SN, Belkaid Y, Kurt-Jones EA, Karp CL: Negative regulation of toll-like receptor 4 signaling by the Toll-like receptor homolog RP105. Nat Immunol 2005;6:571-578.

34 Sakurai H, Tashiro K, Kawabata K, Yamaguchi T, Sakurai F, Nakagawa S, Mizuguchi H: Adenoviral expression of suppressor of cytokine signaling-1 reduces adenovirus vectorinduced innate immune responses. J Immunol 2008; 180:4931-4938.

35 Gavin AL, Hoebe K, Duong B, Ota T, Martin C, Beutler B, Nemazee D: Adjuvant-enhanced antibody responses in the absence of toll-like receptor signaling. Science 2006; 314:1936-1938. 